\title{
Surface Molecular Imprinting by Atom Transfer Radical
}

\section{Polymerization}

Xiaolin Wei, Xiao Li, Scott M. Husson*

Department of Chemical Engineering, Clemson University, Clemson, SC 29634-0909

AUTHOR EMAIL ADDRESS: shusson@ clemson.edu

\section{RECEIVED DATE}

* To whom correspondence can be addressed. Tel.: (864) 656-4502. Fax: (864) 656-0784. E-mail: shusson@clemson.edu.

Supporting Information. Because of its high sensitivity, the fluorescence method quantifies the specific binding of fluorescently-labeled biomolecules to the surface, and also provides an estimate of the surface homogeneity of bound biomolecules. One challenge to perform the quantitative analysis is to prepare the calibration standard. The standard must satisfy at least two requirements. First, the standard sample must cover the surface uniformly without aggregation. Second, the intensity of the fluorescence should be interpretable in terms of the surface density of a specific fluorescent label.

Initial results showed that solution evaporation of the fluorescent molecule onto the surface of micro-well plates used for measurement led to non-uniform aggregation of the fluorescent molecules. By this method, among the 16 positions in one single well, the variation coefficient (calculated by 
dividing the standard deviation by the average) was typically 0.6 to 0.7 . Thus, to develop a calibration plot, an alternate approach was followed. Commercially available fluorescent microspheres are used widely to calibrate ${ }^{1}$; because of their small size, they are transparent to light in aqueous suspensions and behave like a homogeneous solution ${ }^{2}$. In our lab, nanospheres of silica were used as a substrate and dansyl groups were grafted on the surface by two steps (see Scheme 1). The first step reacts surface silanol groups with aminopropyldimethylethoxysilane (APES). The APES has a terminal amine group, which was then reacted with dansyl chloride in a second step. Because of the fluorescent transparency and good aqueous solubility of nanosilica, the grafted molar amount of dansyl group on the silica surface was measurable by fluorometry. The $\mathrm{pH}$ affected the fluorescent intensity of dansyl group dramatically; when $\mathrm{pH}$ was lower than 4 , the amine group was protonated and the fluorescence emission at $510 \mathrm{~nm}$ was decreased dramatically, consistent with the findings of Montalti et al. ${ }^{2}$ Therefore, all calibration experiments were done with $\mathrm{pH}$ controlled at 7. For preparing the calibration standard of solid nano-dansyl-silica, a known mass of dansyl-silica was dissolved in solution with $\mathrm{pH}=7$, and a $5 \mu \mathrm{l}$ drop of this solution was pipetted into the center of each of the 24 wells in the fluorescent microplates. This drop of solution was spread between a coverglass with an area of $1 \mathrm{~cm}^{2}$ and the bottom of well in the microplate; then the sample was allowed to dry in the dark, where light radiation to the sample was avoided. Finally, the fluorescence measurement was performed for the solid sample. This approach produced a uniform distribution of fluorophore; the typical variation coefficient was less than 0.08 . A standard calibration was generated correlating the mass or moles of dansyl group to the fluorescence reading. In order to know the mass of dansyl group per mass of nanoparticles, a second solution-phase calibration was done to correlate dansyl chloride concentration in solution at $\mathrm{pH}=7$ to fluorescence emission intensity. 
Figure 1 shows two calibration plots; one is for dansyl chloride and the other is for dansyl-silica in water. Both plots were obtained by using the same microplates and the same settings for the fluorescence reader. The grafted molar amount of dansyl group on silica was calculated by using these two plots; the grafted density was found to be $38.19 \mu \mathrm{mol}$ of dansyl group per gram of nano-silica.

Figure 2 shows the calibration plots that relate fluorescence emission intensities to areal molar amount of dansyl groups for dansyl-coated silica nanoparticles.

\section{References}

1. Galbraith, W.; Ryan, K.W.; Gliksman, N.; Taylor, D.L.; Waggoner, A.S. Comput. Med. Imag. Graph, 1989, 13, 47-60.

2. Montalti, M.; Prodi, L.; Zaccheroni, N.; Falini, G. J Am. Chem. Soc. 2002, 124, 13540-13546. 
STEP 1

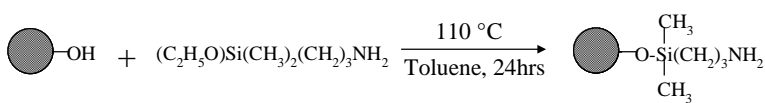

STEP 2

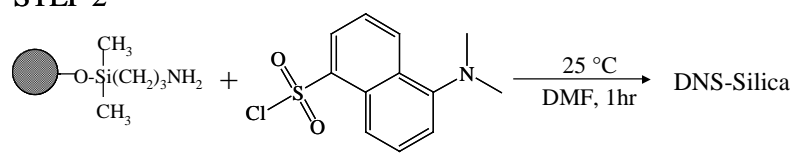

Scheme 1. Synthetic pathway to graft dansyl chloride functional groups on the nano-silica particles. 
Molar Amount of Dansyl Functional Groups, picomoles

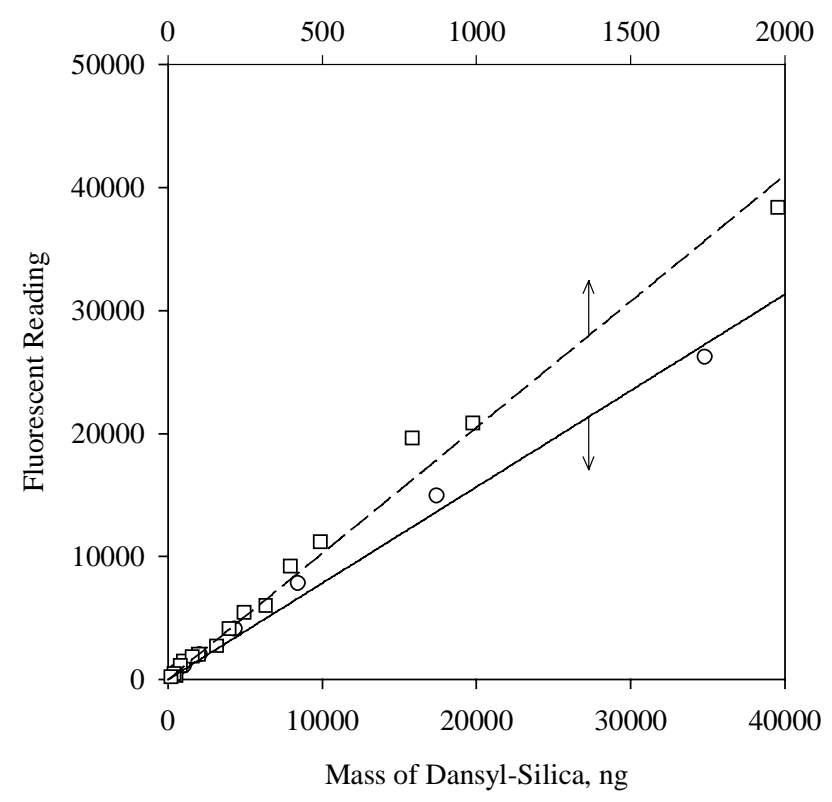

Figure 1. Calibration plots for the dansyl chloride (squares) and nano-dansyl-silica (circles) in aqueous HEPES buffer solution at $\mathrm{pH}=7$. 


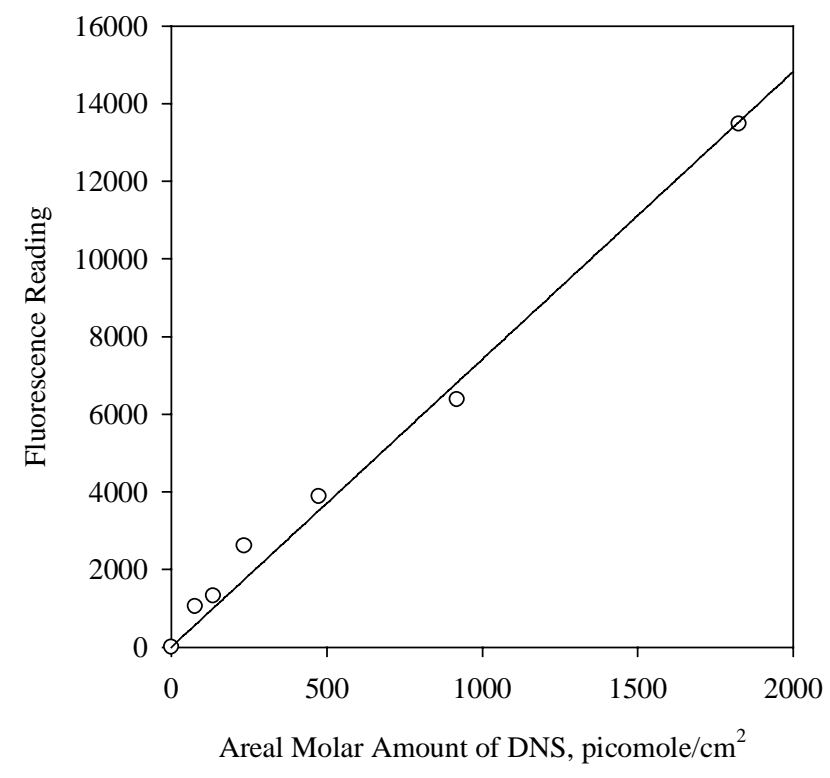

Figure 2. Calibration plot for the nano-dansyl-silica in dry solid adsorbed state. 